\title{
Polyethylene terephthalate tape augmentation as a solution in recurrent quadriceps tendon ruptures
}

\author{
Maciej Leciejewski ${ }^{1, A, C, D}$, Aleksandra Królikowska ${ }^{2, E}$, Paweł Reichert ${ }^{3, C, E, F}$ \\ ${ }^{1}$ Department of Trauma and Orthopedic Surgery, District Hospital, Puszczykowo, Poland \\ ${ }^{2}$ College of Physiotherapy, Wroctaw, Poland \\ ${ }^{3}$ Division of Sports Medicine, Wroclaw Medical University, Poland \\ A - research concept and design; $\mathrm{B}$ - collection and/or assembly of data; $\mathrm{C}$ - data analysis and interpretation; \\ $D$ - writing the article; $E$ - critical revision of the article; $F$ - final approval of the article
}

\section{Address for correspondence}

Maciej Leciejewski

E-mail: leciej@gmail.com

\section{Funding sources \\ None declared}

Conflict of interest

None declared

Received on October 29, 2018

Reviewed on December 9, 2018

Accepted on January 16, 2019
Cite as

Leciejewski M, Królikowska A, Reichert P.

Polyethylene terephthalate tape augmentation as a solution

in recurrent quadriceps tendon ruptures.

Polim Med. 2018;48(1):53-56. doi:10.17219/pim/102977

DOI

10.17219/pim/102977

Copyright

○ 2018 by Wroclaw Medical University

This is an article distributed under the terms of the

Creative Commons Attribution Non-Commercial License

(http://creativecommons.org/licenses/by-nc-nd/4.0/)

\begin{abstract}
Quadriceps tendon rupture is a severe and demanding problem in knee surgery, especially when it is recurrent and when elderly patients are involved. It can have a devastating impact when it is a complication following knee arthroplasty. There are many procedures for dealing with this problem, but none of them offer reliable results. The most popular methods of treatment are traditional transosseous sutures and suture anchors, often in combination with semitendinosus augmentation. In cases of osteoporotic bone or hamstring insufficiency, these solutions are not appropriate. One way to manage quadriceps tendon rupture is to use polyethylene terephthalate tape (poly tape) as scaffolding for tissue ingrowth. Because of its structure, poly tape provides adequate strength and allows early mobilization. Besides being durable, multifilament high tenacity polyethylene terephthalate is flexible. Poly tape augmentation is particularly recommended in the following cases: recurrent rupture of the quadriceps tendon; extensor apparatus damage following total knee arthroplasty (TKA); delayed diagnosis of quadriceps tendon rupture; and in elderly patients (with weak bones and poor ligament quality). The surgical technique is simple and the procedure has a low complication rate. There have been many studies confirming the security of polyethylene terephthalate use in the human body. There is also a great deal of evidence concerning tissue ingrowth in the mesh structure of poly tape. Allergic reactions and inflammatory responses are rare.
\end{abstract}

Key words: quadriceps tendon rupture, poly tapes, ligament augmentation 


\section{Introduction}

The quadriceps tendon attaches all 4 quadriceps femoris muscles (QMFs) to the proximal part of the patella. In more than $50 \%$ of cases, the QMF tendon consists of 3 layers. ${ }^{1}$ The most superficial layer is formed by the rectus femoris muscle, which passes above the patella to form the patellar tendon. The intermediate layer arises from the vastus lateralis and the vastus medialis muscles, which also form the lateral and medial patellar retinacula and play a very important role in patellofemoral joint stability. The deepest layer of the QMF tendon originates from the vastus intermedius muscle. The articularis genus muscle, which makes up the deepest part of the intermedius, is also in this vicinity, ${ }^{2}$ but because it varies greatly in terms of size and number of fibers, it is often not mentioned.

Variations in the structure of the QMF tendon is quite common; it sometimes consists of 4 layers (6\%) or of 2 layers (30\%), and in some cases the layers are hard to identify. The medial and lateral muscles may themselves be 2-ply. The longitudinal fibers may be crossed by oblique portions of the muscles. The patellofemoral ligaments also vary a great deal; the medial patellofemoral ligament (MPFL) is more common than the lateral patellofemoral ligament (LPFL). ${ }^{3}$

The QMF is the largest human muscle and the forces transmitted through its tendon are extremely large. Calculated per square centimeter, its mechanical capacities are very similar to those of the anterior cruciate ligament (ACL). In many studies, the QMF is described as good material for ACL grafting. 4

\section{Epidemiology of and risk factors for quadriceps tendon rupture}

Knee extensor apparatus ruptures as a whole are rare. Quadriceps tendon ruptures, with an incidence of $1.37 / 100,000$, are more common than patellar tendon ruptures $(0.68 / 100,000)$, but much less frequent than patella fractures $(13.1 / 100,000)$. Quadriceps femoris muscles tendon ruptures usually occur in patients over 40 years of age and are much more frequent in males than in females $(8: 1) .^{5}$

The most important risk factors for QMF tendon rupture is overweight (mean BMI $\left.30.0 \pm 6.05 \mathrm{~kg} / \mathrm{m}^{2}\right) .{ }^{5}$ Medical comorbidities are common in tendon ruptures, especially among women. Most frequent are diabetes, rheumatoid arthritis (steroid use), renal failure ${ }^{6}$ and connective tissue disorders. A history of intra-articular injections and the use of statins are also proven risk factors. ${ }^{7}$

The frequency of recurrent QMF tendon rupture is hard to estimate. Few trials have addressed this problem, so data is scarce; most published reports are case studies.

\section{Clinical problems}

Standard techniques used to repair QMF tendon rupture have some disadvantages ${ }^{8,9}$; they lack sufficient strength to permit patients to exercise the full range of motion. There is no consensus regarding rehabilitation protocol. In the past, after QMF tendon repair the knee was immobilized for 6 weeks in full extension, resulting in muscle mass loss, stiffness, limited range of motion, and a very long rehabilitation period after that. On the other hand, although early movement and flexion exercises may improve the rehabilitation process, some authors suggest that a protective cast is necessary. ${ }^{10}$ In elderly patients, especially in recurrent cases, or in patients with tendon ruptures following knee arthroplasty, a long cast and immobility have a negative impact on further treatment. Because of age-related comorbidities, many additional complications may occur (deep vein thrombosis, skin lacerations, muscular dystrophy, joint contracture, stiffness). A better solution is therefore needed. There have been many attempts to use grafts to strengthen the damaged area, including grafts from the semitendinosus (gracilis) tendon ${ }^{11,12}$ or the Achilles tendon. ${ }^{13}$ However, in elderly patients such autografts are weak and morbidity at the donor site may be dangerous. The use of allografts has its own risks (e.g., transmission of infectious diseases) and there are frequent problems with availability. ${ }^{14}$ Nowadays total knee arthroplasty (TKA) is so common that the number of patient suffering from QMF tendon rupture following arthroplasty is increasing. ${ }^{15,16}$

The aim of using polyethylene terephthalate tape (poly tape) is to enhance the ruptured tendon and allow the patient early mobilization. ${ }^{17-21}$ The tape provides intrinsic strength, enabling the tendon to work against tensile forces.

\section{Surgical technique}

The patient is in a supine position and the leg is free, without a tourniquet (to avoid compressing the QMF and shortening the tendon stump). A midline approach is usually recommended. Scar tissue in the area of the injury is debrided, and the proximal part of the patella is refreshed. The QMF is mobilized. In cases of contracture, release may be needed. There are often adhesions that need to be released; sometimes the rectus femoris muscle must be lengthened (by the V-Y technique or Z-plasty). After these preparatory stages, the first step is to pass poly tape under the distal end of the patella (Fig. 1), where it is connected to the patellar tendon. The tape should not be too deep, to avoid joint penetration. It should be securely anchored in the midsubstance of the patellar tendon. The 2 ends of the tape are then brought up and crossed over the kneecap (the anterior aspect of the patella), keeping the tape flat; additional tacking sutures are used to attach the poly tape to the surrounding tissues and to itself at the crossing point. 


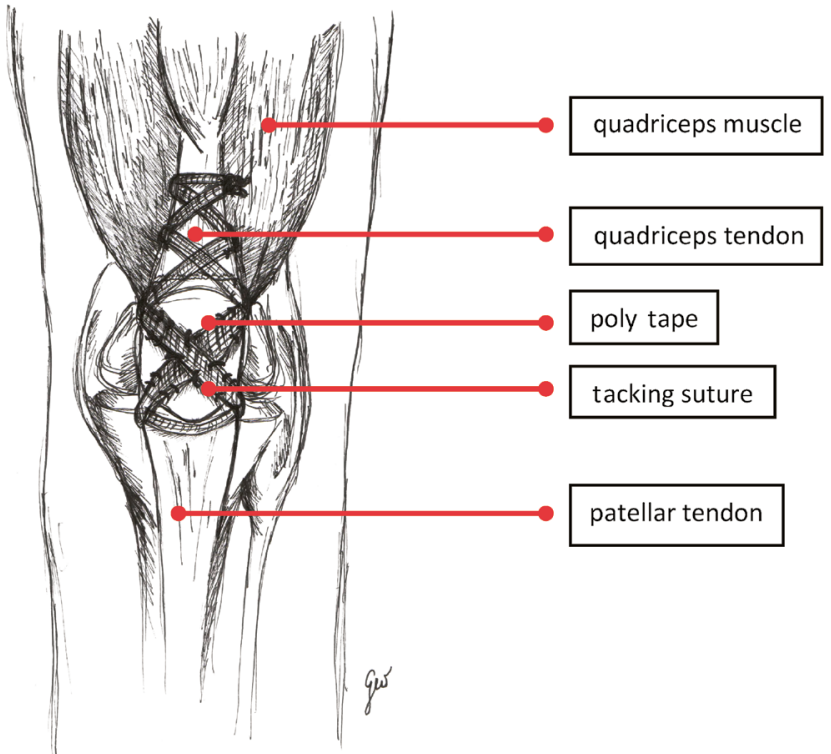

Fig. 1. Schematic poly tape placement on extensor apparatus of the knee (author's own illustration)

The distal end of the quadriceps tendon must be approximate to the patella, and then the tape is passed through the tendon as in a Bunnell suture. Because of the length of the tape, $(30 \times 800 \mathrm{~mm})$, the number of loops is rather arbitrary (Fig. 2). To prevent secondary ischemic damage, it is essential to ensure that the tape is not too tight; the stability of the extensor apparatus should be achieved through the appropriate placement of additional sutures joining the tape and tendon/muscle tissue rather than by putting pressure on the tendon with the tape. Finally, the proximal ends of the tape are knotted and hidden in the muscle. A protective suture is added to avoid knot failure. After that the tape should smoothly interlace the tendon, and any unevenness should be flattened by sutures. It is good to make sure knee flexion in the range of $0-60^{\circ}$ is safe, causing no discontinuity of the extensor apparatus. The wound is closed in the standard way.

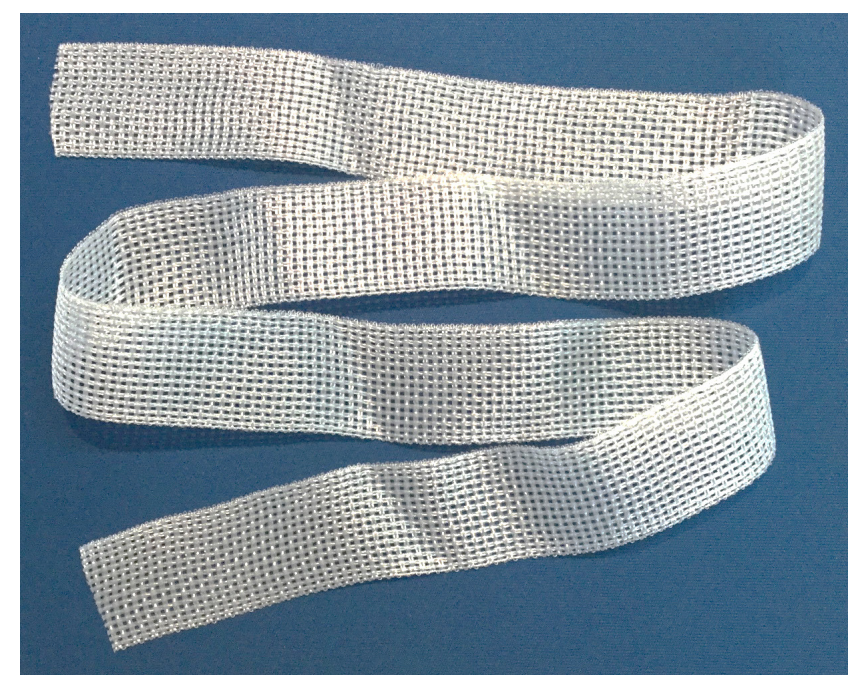

Fig. 2. Poly tape, 30 mm x 800 mm (photo courtesy of Komak sp. z o.o.)

\section{Rehabilitation protocol}

Despite augmentation of the extensor mechanism with poly tape, the rehabilitation protocol is cautious and must be adapted to suit each patient. The use of a cast is not advised; we prefer a brace with an adjustable range of motion. Just after surgery, the brace is fixed in full extension for 2 weeks. During this period the patient is taught how to exercise the QMF isometrically. Walking distances are not limited, but the patient uses 2 crutches. After 2 weeks of immobilization, the range of motion is increased gradually by $30^{\circ}$ of flexion per 2 -week periods $\left(0-30^{\circ}\right.$ : 2-4 weeks; $0-60^{\circ}$ : 4-6 weeks; $0-90^{\circ}$ : 6-8 weeks). The patient is advised to try to use 1 crutch in the opposite hand at this stage. Movement is achieved passively; active flexion of the knee is allowed 6 weeks after the reconstructive procedure. Active extension is initiated after 8 weeks. The brace is no longer needed. Strengthening of the quadriceps muscle continues, along with gait training. An important part of the protocol is the reinforcement of the proximal and core musculature (gluteal muscles, paraspinal muscles and abdominal muscles). Proprioception and balance training are also crucial. Sometimes an imbalance between the lateral and medial stabilizers of the patella may occur. Special care must be taken of vastus medialis obliquus (VMO), which is naturally the smallest part of the quadriceps and it frequently atrophies after an injury. Training the VMO is essential for proper patellofemoral tracking and painless flexion. Patellar mobilization is done throughout the rehabilitation process and patients are taught how to do it by themselves. ${ }^{22}$

\section{Discussion}

Because of aging societies and the increasing number of degenerative diseases, there are new demands on modern medicine. There is a need to look for materials that provide good structural support and are safe in widespread use. Along with metallic and ceramic materials, polymers play a key role. ${ }^{23}$ Polymers are used as acrylic bone cements (to anchorage prostheses to the bone), as polyethylene inserts in acetabular cups in hip prostheses or as silicone elastomers that replace small joints. The next generation of polymers might be bioabsorbable and useful for bone and other tissue defects. In ligament diseases and discontinuity, a material is required that acts as 3-dimensional scaffold. Poly tape is made of woven multifilament high-tenacity polyester fiber. The open-weave structure acts as a matrix and leaves space for tissue ingrowth. The parallel fibers provide a high degree of strength. The mesh is made of pure polyethylene terephthalate (PET), without any additions. It consists of repeating units of the monomer ethylene terephthalate $\left(\mathrm{C}_{10} \mathrm{H}_{8} \mathrm{O}_{4}\right)$. Its physical properties are as follows: tensile strength $55-75 \mathrm{MPa}$; elastic limit 50-150\%; glass transition temperature $67-81^{\circ} \mathrm{C}$, 
with a softening point of $265^{\circ} \mathrm{C}$. It is lightweight, strong and impact-resistant, as well as hygroscopic (absorbing water from its surroundings).

Years of experience with similar polymers provide reproducible results. Long-term outcomes in strengthening the abdominal wall in hernias are good or even excellent. ${ }^{24}$ The infection rate is very low and recurrence is rare. Hypersensitivity to implant materials occurs infrequently. That is why use of tape in tendon/muscle injury seems to be safe. There is no data suggesting heterotopic ossification or muscle fibrosis. In comparison to other techniques, it offers the possibility of early movement and immediate isometric exercises. Reconstruction of the extensor apparatus with poly tape is worth considering in difficult recurrent cases.

The use of poly tape in patellar tendon rupture is also promising. The technique is similar to what has been described above, likewise reproducible and not difficult. It can be even applied when the extensor apparatus needs to be reconstructed following patellectomy.

In the future, poly tape may be applied in the reconstruction of other tendons, i.e., in the biceps brachii tendon or the Achilles tendon. Because of good clinical results, the use of poly tapes in ligament surgery is likely to gain popularity.

\section{ORCID iDs}

Maciej Leciejewski (1) https://orcid.org/0000-0002-2081-8788 Aleksandra Królikowska (1) https://orcid.org/0000-0002-6283-5500 Paweł Reichert (1) https://orcid.org/0000-0002-0271-4950

\section{References}

1. Zeiss J, Saddemi SR, Ebraheim NA. MR imaging of the quadriceps tendon: Normal layered configuration and its importance in cases of tendon rupture. AJR Am J Roentgenol. 1992;159(5):1031-1034.

2. Grob K, Gilbey H, Manestar M, Ackland T, Kuster MS. The anatomy of the articularis genus muscle and its relation to the extensor apparatus of the knee. JBJS Open Access. 2017;2(4):e0034.

3. Waligora AC, Johanson NA, Hirsch BE. Clinical anatomy of the quadriceps femoris and extensor apparatus of the knee. Clin Orthop Relat Res. 2009;467(12):3297-3306.

4. Hurley ET, Calvo-Gurry M, Withers D, Farrington SK, Moran R, Moran CJ. Quadriceps tendon autograft in anterior cruciate ligament reconstruction: A systematic review. Arthroscopy. 2018;34(5):1690-1698.

5. Garner MR, Gausden E, Berkes MB, Nguyen JT, Lorich DG. Extensor mechanism injuries of the knee: Demographic characteristics and comorbidities from a review of 726 patients records. J Bone Joint Surg Am. 2015;97(19):1592-1596.

6. Lim CH, Landon KJ, Chan GM. Bilateral quadriceps femoris tendon rupture in a patient with chronic renal insufficiency: A case report. J Emerg Med. 2016;51(4):e85-e87.

7. Deren ME, Klinge SA, Mukand NH, Mukand JA. Tendinopathy and tendon rupture associated with statins. JBJS Rev. 2016;4(5). doi:10.2106/JBJS.RVW.15.00072

8. Plesser S, Keilani M, Vekszler G, et al. Clinical outcomes after treatment of quadriceps tendon ruptures show equal results independent of suture anchor or transosseous repair technique used: A pilot study. PLoS One. 2018;13(3):e0194376.

9. Brossard P, Le Roux G, Vasse B; Orthopedics, Traumatology Society of Western France (SOO). Acute quadriceps tendon rupture repaired by suture anchors: Outcomes at 7 years' follow-up in 25 cases. Orthop Traumatol Surg Res. 2017;103(4):597-601.
10. Boudissa M, Roudet A, Rubens-Duval B, Chaussard C, Saragaglia D. Acute quadriceps tendon ruptures: $A$ series of 50 knees with an average follow-up of more than 6 years. Orthop Traumatol Surg Res. 2014;100(2):213-216.

11. Chahla J, DePhillipo NN, Cinque ME. Open repair of quadriceps tendon with suture anchors and semitendinosus tendon allograft augmentation. Arthrosc Tech. 2017;6(6):e2071-e2077.

12. Maffuli N, Papalia R, Torre G, Denaro V. Surgical treatment for failure of repair of patellar and quadriceps tendon rupture with ipsilateral hamstring tendon graft. Sports Med Arthrosc Rev. 2017;25(1):51-55.

13. Wise BT, Erens G, Pour AE, Bradbury TL, Roberson JR. Longterm results of extensor mechanism reconstruction using Achilles tendon allograft after total knee arthroplasty. Int Orthop. 2018;42(10):2367-2373.

14. Ricciardi BF, Oi K, Trivellas M, Lee YY, Della Valle AG, Westrich GH. Survivorship of extensor mechanism allograft reconstruction after total knee arthroplasty. J Arthroplasty. 2017;32(1):183-188.

15. Chhapan J, Sankineani SR, Chiranjeevi T, Reddy MV, Reddy D, Gurava Reddy AV. Early quadriceps tendon rupture after primary total knee arthroplasty. Knee. 2018;25(1):192-194.

16. Soong JW, Silva AN, Andrew TH. Disruption of quadriceps tendon after total knee arthroplasty: Case report of four cases. $J$ Orthop Surg (Hong Kong). 2017;25(2):2309499017717206.

17. Abdel MP, Salib CG, Mara KC, Pagnano MW, Perry KI, Hanssen AD. Extensor mechanism reconstruction with Ue of Marlex mesh: A series study of 77 total knee arthroplasties. J Bone Joint Surg Am. 2018;100(15):1309-1318.

18. Paci JM, Pawlak A. Knotless tape suture fixation of quadriceps tendon rupture: A novel technique. Am J Orthop (Belle Mead NJ). 2018;47(1). doi:10.12788/ajo.2018.0002

19. Nodzo SR, Rachala SR. Polypropylene mesh augmentation for complete quadriceps rupture after total knee arthroplasty. Knee. 2016;23(1):177-180.

20. Morrey MC, Barlow JD, Abdel MP, Hanssen AD. Synthetic mesh augmentation of acute and subacute quadriceps tendon repair. Orthopedics. 2016;39(1):e9-13.

21. Rehman H, Kovacs P. Quadriceps tendon repair using hamstring, prolene mesh and autologous conditioned plasma augmentation: A novel technique for repair of chronic quadriceps tendon rupture. Knee. 2015;22(6):664-668.

22. Langenhan R, Baumann M, Ricart P. Postoperative functional rehabilitation after repair of quadriceps tendon ruptures: A comparison of two different protocols. Knee Surg Sports Traumatol Arthrosc. 2012;20(11):2275-2278.

23. Navarro M, Michiardi A, Castano O, Planell JA. Biomaterials in orthopedics. JR Soc Interface. 2008;5(27):1137-1158.

24. Gordon AC, Gillespie C, Son J, Polhill T, Leibman S, Smith GS. Longterm outcomes of laparoscopic large hiatus hernia repair with nonabsorbable mesh. Dis Esophagus. 2018;31(5). doi:10.1093/dote/dox156 